\title{
Extending the Operating Distance of Inductive Proximity Sensor Using Magnetoplated Wire
}

\author{
Tsutomu Mizuno ${ }^{1}$, Takahiro Mizuguchi ${ }^{1}$, Yusuke Isono ${ }^{1}$, Takayuki Fujii ${ }^{1}$, Yoshio Kishi ${ }^{2}$, Katsuhiko Nakaya ${ }^{2}$, \\ Masaki Kasai ${ }^{2}$, and Atsushi Shimizu ${ }^{2}$ \\ ${ }^{1}$ Faculty of Engineering, Shinshu University, Nagano 380-8553, Japan \\ ${ }^{2}$ Koyo Electronics Industries Co., Ltd. Yamanashi 409-1501, Japan
}

\begin{abstract}
Inductive proximity sensors are noncontact sensing devices used to detect the approach of a target by an increase in coil resistance due to eddy current loss. Extending the operating distance of these sensors is demanded. In this paper, we propose the use of a magnetoplated wire (MPW) as a sensing coil. The MPW is a copper wire, whose circumference is plated with a magnetic thin film. We analyze the impedance of a proximity sensor using a copper wire (COW) and MPW coils by a finite element method. The use of the MPW results in a decrease in $\mathrm{AC}$ resistance due to the proximity effect, an increase in inductance, and the generation of a higher flux than when the COW is used. Therefore, it is possible to increase the quality factor $Q$ of the MPW coil. As a result, the operating distances of the MPW and COW coils are 5.0 and $3.8 \mathrm{~mm}$, respectively. The operating distance of the MPW coil is 1.3-fold that of the COW coil.
\end{abstract}

Index Terms-Coil, copper wire, finite element method, inductive proximity sensor, magnetoplated wire, operating distance, output voltage, quality factor.

\section{INTRODUCTION}

I NDUCTIVE proximity sensors (proximity sensors) are noncontact sensing devices used to detect the approach of a target by changes in coil impedance and quality factor $Q$ due to eddy current loss [1]. The conventional proximity sensor has a short operating distance. Accordingly, the target collides with this sensor, causing damage to the sensor. Therefore, extending the operating distance of this sensor is demanded.

The quality factor $Q$ of the proximity sensor was analyzed by a finite element method (FEM) [1], [2]. However, designs of the coil and the improvement in $Q$ for extending the operating distance have not yet been accomplished.

Therefore, we propose the use of a magnetoplated wire (MPW) as the sensing coil to extend the operating distance. The MPW is a copper wire (COW) whose circumference is plated with a magnetic thin film. The MPW coil decreases the $\mathrm{AC}$ resistance due to the proximity effect and increases the inductance [3]-[5].

In this study, we examine a proximity sensor using COW and MPW coils both experimentally and analytically by FEM to extend the operating distance. The following points concerning the proximity sensor with the MPW are discussed.

1) Requirement for extension of operating distance

2) Extension of operating distance using MPW

\section{REQUiREMENT FOR EXTENSION OF OPERATING DistANCE}

Fig. 1 shows the detection principle of an inductive proximity sensor. The impedance and $Q$ of the proximity sensor vary with the distance $s$ between the sensing part (coil and ferrite core) and the target (cold rolling carbon steel sheet). In this proximity

Manuscript received March 06, 2009; revised April 22, 2009. Current version published September 18, 2009. Corresponding author: M. Takahiro (e-mail: t08a248@ shinshu-u.ac.jp).

Color versions of one or more of the figures in this paper are available online at http://ieeexplore.ieee.org.

Digital Object Identifier 10.1109/TMAG.2009.2021855

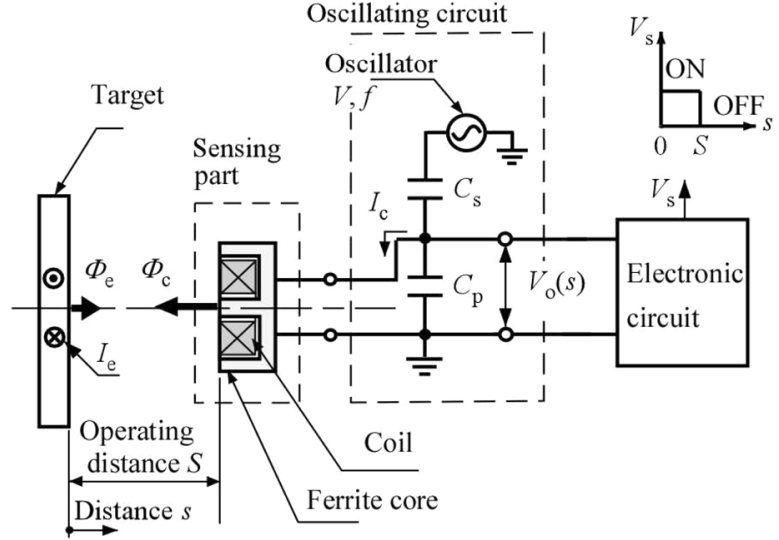

Fig. 1. Detection principle of inductive proximity sensor (coil: $N=100$ turns, ferrite core: Tomita Electric Co., Ltd. $2 \mathrm{H} 6-\mathrm{PC}-7 \times 2.1$, target: cold rolling carbon steel sheet, $1 \times 20 \times 20 \mathrm{~mm}^{3}$ ).

sensor, the change in $Q$ is converted into an output voltage $V_{\mathrm{o}}$ in the oscillating circuit. The electric circuit outputs the proximity sensor output $V_{\mathrm{s}}$ of the ON/OFF operation depending on the change in output voltage $V_{0}$. The distance from the surface of the sensing part to the outside of the body of the target when the proximity sensor output $V_{\mathrm{s}}$ is turned on and off is called the operating distance $S$.

The relationship between the $Q$ of the proximity sensor $(Q(s))$ and the output voltage $V_{\mathrm{o}}\left(k=1+C_{\mathrm{p}} / C_{\mathrm{s}} \gg 1\right)$ is

$$
\begin{aligned}
V_{\mathrm{o}}(s) & \approx \frac{Q(s)}{k} \cdot V=\frac{\omega L(s)}{k R(s)} \cdot V \\
& =\frac{\omega L(s)}{k\left\{R_{\mathrm{dc}}+R_{\mathrm{s}}+R_{\mathrm{p}}+R_{\mathrm{c}}+R_{\mathrm{t}}(s)\right\}} \cdot V(\mathrm{~V})
\end{aligned}
$$

where $s$ is the distance $(\mathrm{m}), \omega$ is the angular frequency $(=2 \pi f$, $\mathrm{rad} / s), f$ is the exciting frequency $(\mathrm{Hz}), L(s)$ is the inductance $(\mathrm{H}), R(s)$ is the resistance $(\Omega), R_{\mathrm{dc}}$ is the $\mathrm{DC}$ resistance $(\Omega), R_{\mathrm{S}}$ is the resistance $(\Omega)$ due to the skin effect, $R_{\mathrm{p}}$ is the resistance $(\Omega)$ due to the proximity effect, $R_{\mathrm{c}}$ is the resistance $(\Omega)$ due to the core loss, $R_{\mathrm{t}}(s)$ is the resistance $(\Omega)$ due to the target eddy 


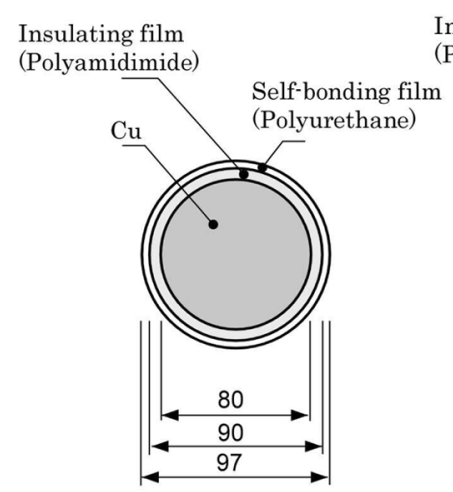

(a)
Insulating film

(Polyamidimide)

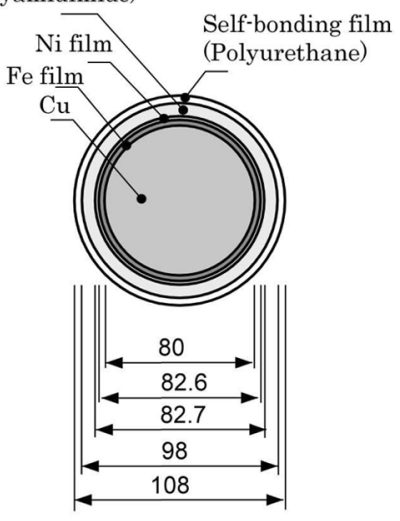

(b)

Fig. 2. Structures of COW and MPW (unit: $\mu \mathrm{m}$ ). (a) COW. (b) MPW

current loss, $V$ is the exciting voltage $(\mathrm{V}), C_{\mathrm{p}}$ is the parallel capacitance $(\mathrm{F})$, and $C_{\mathrm{s}}$ is the series capacitance $(\mathrm{F})$.

Under the $k \gg 1$ condition, $V_{\mathrm{o}}$ is proportional to the $Q$ of the coil. Therefore, it is essential to improve the $Q$ of the coil to extend the operating distance [4]. The following equation is obtained by differentiating (1) with respect to the distance $s$,

$$
\begin{aligned}
\frac{\mathrm{d} V_{\mathrm{o}}(s)}{\mathrm{d} s} \approx & \frac{\mathrm{d} Q(s)}{k \mathrm{~d} s} \cdot V \\
= & \frac{-\omega L}{k \cdot\left\{R_{\mathrm{dc}}+R_{\mathrm{s}}+R_{\mathrm{p}}+R_{\mathrm{c}}+R_{\mathrm{t}}(s)\right\}^{2}} \\
& \cdot \frac{\mathrm{d}}{\mathrm{d} s} R_{\mathrm{t}}(s) \cdot V \quad(\mathrm{~V} / \mathrm{m}) .
\end{aligned}
$$

Equation (2) shows that the stable ON/OFF operation in the electronic circuit is attained by an increase in $\mathrm{d} Q(s) / \mathrm{d} s$. As a result, the operating distance $S$ is extended.

Fig. 2 shows the structures of the COW and MPW. The MPW is a copper wire plated with magnetic thin films ( $\mathrm{Fe}$ and $\mathrm{Ni}$ ) [3]. The thicknesses of the Fe and Ni thin films are 1.3 and $0.05 \mu \mathrm{m}$, respectively, as shown in Fig. 2(b). The Ni film is plated for ease of soldering.

Fig. 3 shows the $B-H$ loop of the MPW. We measured the $B$ - $H$ loop of the MPW of $10 \mathrm{~mm}$ length with a vibrating sample magnetometer (Riken Denshi BVH-55). The magnetic field affected the MPW longitudinally. In this study, the MPW was affected by the magnetic field of $\pm 80 \mathrm{~A} / \mathrm{m}$. We assumed the following matter.

i) The Fe thin film is isotropic.

ii) The diamagnetic field is disregarded because the demagnetizing factor of the MPW with $80 \mu \mathrm{m}$ diameter and $10 \mathrm{~mm}$ length is $10^{-3}$ or less.

The relative permeability of the MPW, $\mu_{\mathrm{r}}$, was 80 , as shown in Fig. 3.

\section{COMPARISON BETWEen COW AND MPW COILS IN TERMS OF QUALITY FACTOR $Q$}

Table I shows a list of conditions for FEM analysis. We analyzed models of the sensing parts using the JMAG-Studio (x64) Ver. 9.0 FEM software (the axisymmetric version of transient

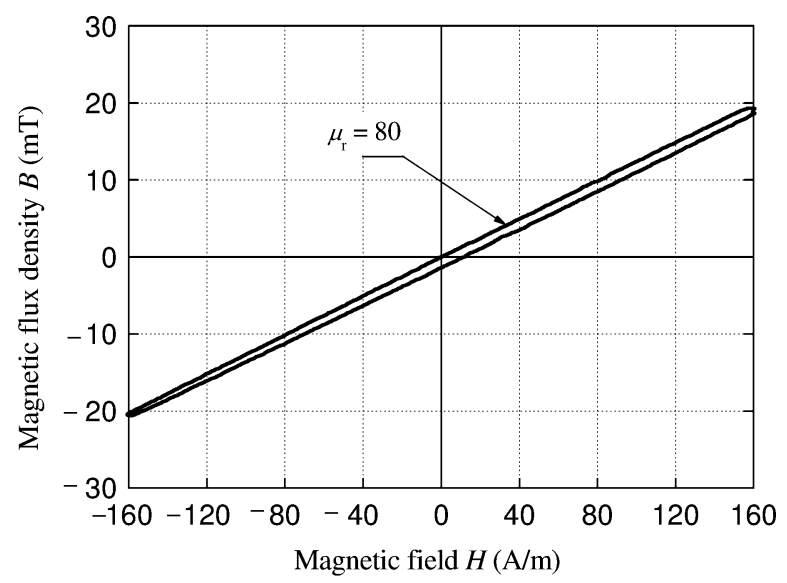

Fig. 3. $B-H$ loop of MPW (measured with Riken Denshi BVH-55).

\begin{tabular}{|c|c|}
\hline Item & Content \\
\hline Software & JMAG-Studio (x64) Ver. 9.0 \\
\hline Analysis condition & $\begin{array}{l}\text { Axisymetric version of transient } \\
\text { magnetic } \\
\text { (considered eddy current) }\end{array}$ \\
\hline Frequency range & $100 \mathrm{k}-1 \mathrm{M}(\mathrm{Hz})$ \\
\hline Current setting & $I=1(\mathrm{~mA})$ \\
\hline Material properties & $\begin{array}{l}\mathrm{Cu}\left(\rho=0.0172 \mu \Omega \mathrm{m}, \mu_{\mathrm{r}}=0.999991\right) \\
\text { Fe and Ni thin film }\left(\rho=0.098 \mu \Omega \mathrm{m}, \mu_{\mathrm{r}}\right. \\
=80) \\
\text { Target: cold rolling carbon steel sheet }(\rho \\
\left.=0.14 \mu \Omega \mathrm{m}, \mu_{\mathrm{r}}=289\right) \\
\text { Ferrite: Tomita Electric Co., Ltd., } 2 \mathrm{H} 6 \\
\left\{\rho=2.3 \Omega \mathrm{m}, \mu^{\prime}=1200, \mu^{\prime \prime}=40 \text { (at } 1\right. \\
\mathrm{MHz})\}\end{array}$ \\
\hline
\end{tabular}

TABLE I

ANALYSIS CONDITIONS OF FEM

magnetic) [6]. In the FEM analysis model, the structure of the coil ( $N=100$ turns) was reproduced to analyze $R_{\mathrm{p}}$. Furthermore, the sizes of the meshes of the Fe thin films considered affected the skin depth $\delta$ of $15 \mu \mathrm{m}(f=1 \mathrm{MHz})$ for Fe used to analyze the eddy current that acted on a Fe thin film, and mesh size was considered to be $0.4 \mu \mathrm{m}$. The Ni thin film was not considered in the FEM because of its thin thickness. The total number of elements was 2000000 . The relative permeability $\mu_{\mathrm{r}}$ of 80 the MPW measured with the vibrating sample magnetometer was input. The resistivity $\rho$ and relative permeability $\mu_{\mathrm{r}}$ of the cold rolling carbon steel sheet were measured using the samples, whereas the $\rho$ and $\mu_{\mathrm{r}}$ of the ferrite core used were obtained from a list of values in a catalogue.

Fig. 4 shows the impedance vs. frequency characteristics of the COW and MPW sensing parts. We found that the increase in $R$ for the frequency depended on $R_{\mathrm{p}}$ for the following reasons, as shown in Fig. 4(a).

First, $R(\infty)=R_{\mathrm{dc}}+R_{\mathrm{S}}+R_{\mathrm{p}}+R_{\mathrm{c}}$, where $R_{\mathrm{dc}}$ is constant and does not depend on the frequency. Second, the skin depth $\delta$ is $74.7 \mu \mathrm{m}$ for the COW ( $f=780 \mathrm{kHz}$ ). Because the skin depth of the COW is larger than its radius of $40 \mu \mathrm{m}$, the resistance $R_{\mathrm{S}}$ due to the skin effect is considered to be $R_{\mathrm{S}} \approx 0$. Third, the resistance $R_{\mathrm{c}}$ due to the core loss is considered to be zero because the core loss resistance $R_{\mathrm{c}}$ is determined to be $0.05 \Omega$ 


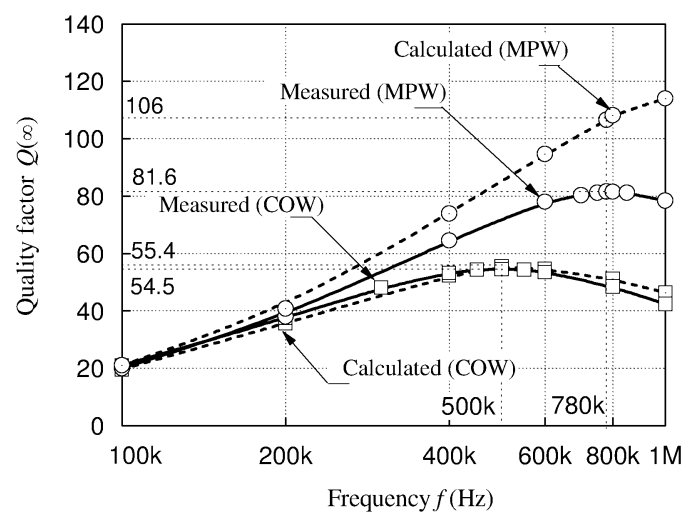

(a)

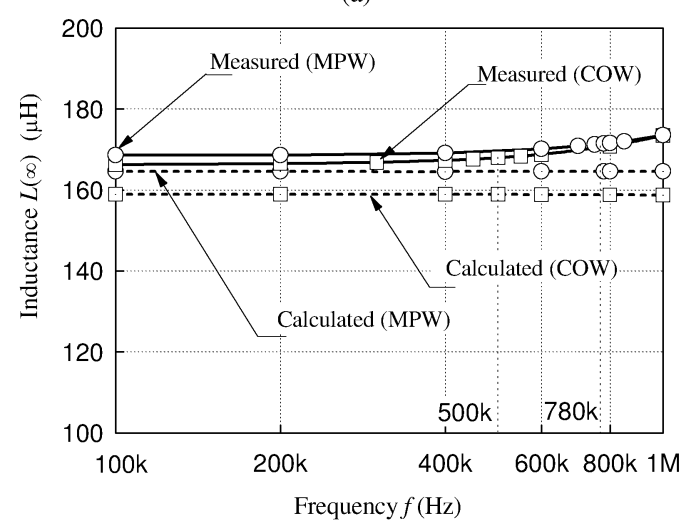

(b)

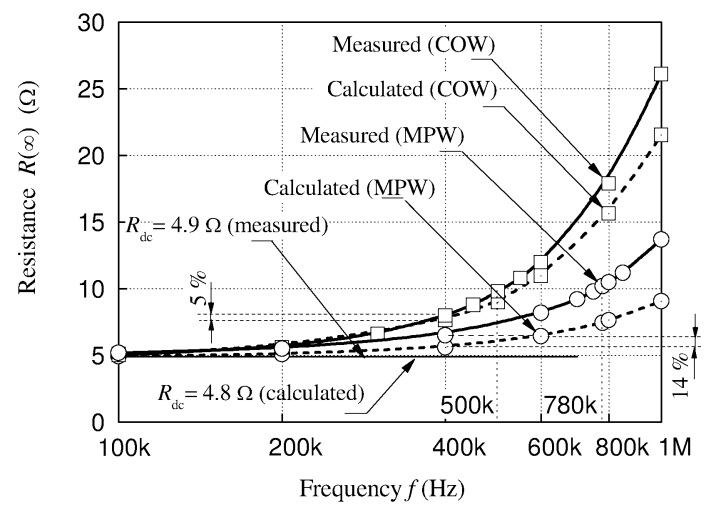

(c)

Fig. 4. Impedance versus frequency characteristics of COW and MPW sensing parts (measured with impedance analyzer HP 4192A). (a) Resistance. (b) Inductance. (c) Quality factor.

in a ferrite core from the FEM analysis result ( $f=780 \mathrm{kHz}$ ). Therefore, the increase in $R$ for the frequency depends on the resistance $R_{\mathrm{p}}$ due to the proximity effect.

The resistances of the COW and MPW at the frequency $f$ of $780 \mathrm{kHz}$ were 16.5 and $10.2 \Omega$, respectively; thus, the resistance of the MPW decreased by $38 \%$ compared with that of the COW. Flux was hardly observed in the conducting wire owing to the effect of the Fe thin film for the MPW compared with the COW, and the MPW is hard to produce the eddy current in the copper moiety. Therefore, the proximity effect was restrained in the MPW, and $R_{\mathrm{p}}$ decreased [4], [5]. As a result, the resistance $R$ of the MPW decreased more markedly than that of the COW.

The calculation errors of the resistances of the COW and MPW at the frequency $f$ of $500 \mathrm{kHz}$ were $5 \%$ and $14 \%$, respec-

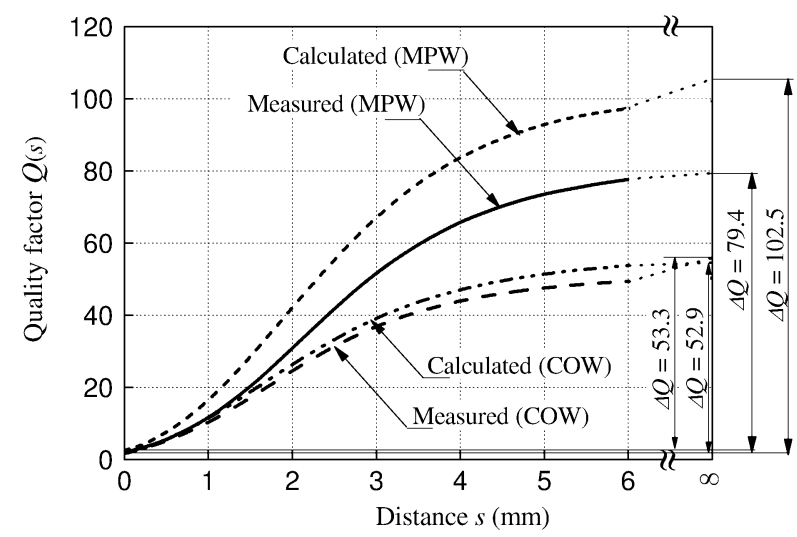

Fig. 5. Quality factor vs. distance characteristics of COW and MPW (COW: $f=500 \mathrm{kHz}$, MPW: $f=780 \mathrm{kHz}$, measured with impedance analyzer HP 4192A).

tively; thus, the calculation error of the MPW was larger than that of the COW. The calculation error of the MPW was caused by the stress affecting the $\mathrm{Fe}$ thin film during coil reeling and the decrease in $\mu_{\mathrm{r}}$.

In addition, in the MPW, $L$ was increased by the Fe thin film, as shown in Fig. 4(b). Furthermore, owing to the decrease in $R$ and the increase in $L$ in the MPW, the $Q$ of the MPW (81.6: $f=780 \mathrm{kHz}$ ) was 1.5 -fold that of the COW (54.5: $f=500 \mathrm{kHz}$ ), as shown in Fig. 4(c). The maxima of $Q$ occurred. $Q=\omega L / R$ where $L$ were constant and dose not depend on the frequency and $\omega L$ were proportional to the frequency. When frequency was high, $R$ suddenly increases due to proximity effect, as shown in Fig. 4(a). Therefore, the maxima of $Q$ appeared, as shown in Fig. 4(c).

\section{EXTENSION OF OPERATING DistANCE}

Fig. 5 shows the $Q$ vs. distance characteristics of the COW and MPW. The measured and FEM values were analyzed at the frequencies (COW: $500 \mathrm{kHz}, \mathrm{MPW}: 780 \mathrm{kHz}$ ) at which the $Q$ of each coil was maximized, as shown in Fig. 4(c). The obtained values were approximated using polynomials of six degrees. The data points and the approximated curves were corresponding because the coefficients between the data points and the approximated curves were more than 0.999. Owing to the decrease in $R$ and the increase in $L$ in the MPW, the $Q$ of the MPW was larger than that of the COW. The change in $Q$ for the MPW $\Delta Q(=Q(\infty)-Q(0))$ was 79.4 , which is 1.5 -fold that of $\Delta Q=53.3$ for the COW.

Fig. 6 shows the comparison of the resistance $R_{\mathrm{t}}$ between the COW and MPW (at $s=3.8 \mathrm{~mm}$ ). It is a resistance ingredient to occur to the COW, MPW and the target in operating distance $S=3.8 \mathrm{~mm}$ of COW. $R_{\mathrm{t}}$ due to the target eddy current loss was derived using

$$
R_{\mathrm{t}}(s)=R(s)-R(\infty) \quad(\Omega) .
$$

The resistances $R$ of the COW and MPW at $s=3.8 \mathrm{~mm}$ were $11.4 \Omega$ and $13.2 \Omega$, respectively. The resistances $R_{\mathrm{t}}$ of the COW and MPW were $1.6 \Omega$ and $3.4 \Omega$, respectively, and the $R_{\mathrm{t}}$ of the MPW was 2.1-fold that of the COW. The resistance $R_{\mathrm{t}}$ due to the target eddy current loss is proportional to square of the 


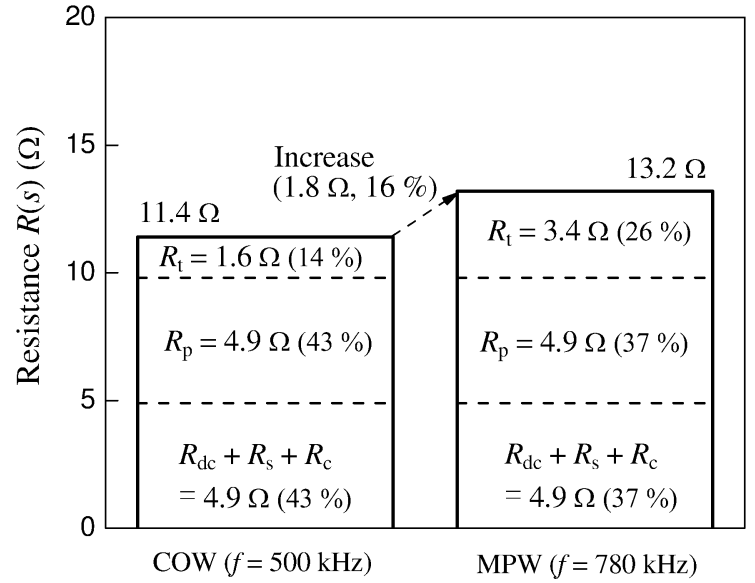

Fig. 6. Comparison of resistance $R_{\mathrm{t}}$ between COW and MPW (at $s=3.8 \mathrm{~mm})$.

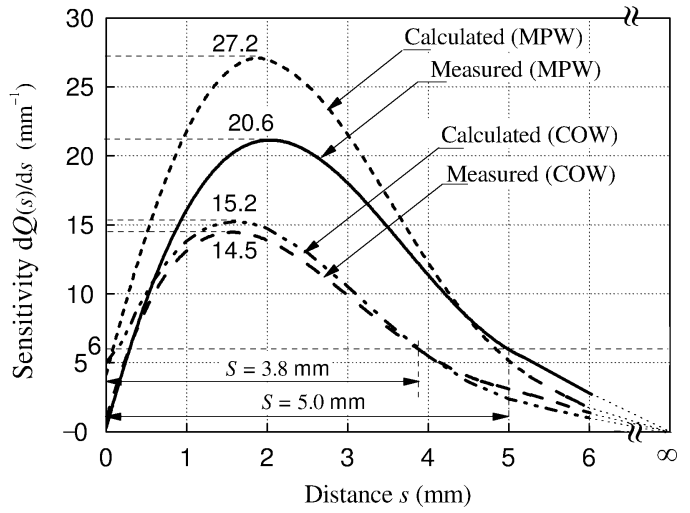

Fig. 7. Sensitivity versus distance characteristics (COW: $f=500 \mathrm{kHz}$, MPW: $f=780 \mathrm{kHz}$, measured with impedance analyzer HP 4192A).

exciting frequency $f$ and flux $\Phi_{\mathrm{c}}$ to reach the target. Therefore the $R_{\mathrm{t}}$ of the MPW was larger than that of the COW.

Fig. 7 shows the sensitivity vs. distance characteristics of the COW and MPW obtained by differentiating the approximated curves shown in Fig. 5. A conventional proximity sensor using the COW operates by ON/OFF switching when $\mathrm{d} Q(s) / \mathrm{d} s=$ $6 \mathrm{~mm}^{-1}$. The operating distance was $5.0 \mathrm{~mm}$ for the MPW, which is 1.3 -fold the value of $S=3.8 \mathrm{~mm}$ for the COW, as shown in Fig. 7.

The extension of the operating distance $S$ was confirmed by improving $Q$, as shown in the (1) and (2). Owing to the decrease in $R_{\mathrm{p}}$ and the increase in $L$ in the MPW, the operating distance $S$ of the MPW was longer than that of the COW.

\section{CONCLUSION}

In this paper, we propose the use of an MPW as the sensing coil of a proximity sensor to extend the operating distance. The following is a summary of our findings.

\section{A. Requirement for Extension of Operating Distance}

We showed that it was essential to improve the $Q$ of the coil to extend the operating distance. In other words, it is necessary to extend the operating distance by reducing the resistance and increasing the inductance of a proximity sensor. The $Q$ values of the MPW ( $f=780 \mathrm{kHz})$ and COW $(f=500 \mathrm{kHz})$ were 54.5 and 81.6, respectively. The $Q$ of the MPW was 1.5 -fold that of the COW.

\section{B. Extension of Operating Distance Using MPW}

The operating distance was $5.0 \mathrm{~mm}$ for the MPW, which is 1.3-fold the value of $S=3.8 \mathrm{~mm}$ for the COW. Owing to the decrease in $R_{\mathrm{p}}$ and the increase in $L$ in the MPW, the operating distance $S$ of the MPW was longer than that of the COW.

\section{REFERENCES}

[1] K. Koibuchi, K. Sawa, T. Honma, T. Hayashi, K. Ueda, and H. Sasaki, "Eddy-current type proximity sensor with closed magnetic circuit geometry," IEEE Trans. Magn., vol. 43, no. 4, pp. 1749-1752, Apr. 2007.

[2] K. D. Anim-Appiah and S. M. Riad, "Analysis and design of ferrite core for eddy-current-killed oscillator inductive proximity sensors," IEEE Trans. Magn., vol. 33, no. 3, pp. 2274-2281, May 1997.

[3] S. Yoshimura, S. Yoshihara, T. Shirakashi, E. Sato, and K. Ishii, "Characteristics of high- $Q$ coils composed of Fe-plated $\mathrm{Cu}$ wire," in Proc. Magneto-Electronics Int. Symp., 1994, pp. 485-487.

[4] T. Mizuno, S. Enoki, T. Hayashi, and H. Shinagawa, "Extending the linearity range of eddy-current displacement sensor with magnetoplated wire," IEEE Trans. Magn., vol. 43, no. 2, pp. 543-548, Feb. 2007.

[5] T. Mizuno, S. Enoki, T. Asahina, T. Suzuki, M. Noda, and H. Shinagawa, "Reduction of proximity effect in coil using magnetoplated wire," IEEE Trans. Magn., vol. 43, no. 6, pp. 2654-2656, Jun. 2007.

[6] “JMAG-Studio Version 9.0 User'S Manual," Japan Research Institute Co., Ltd. 